\title{
Coherent radiation sources based on laser driven plasma waves
}

\author{
D.A. Jaroszynski ${ }^{1}$, B. Ersfeld ${ }^{1}$, M.R. Islam ${ }^{1}$, E. Brunetti ${ }^{1}$, R.P. Shanks ${ }^{1}$, P.A. Grant ${ }^{1}$, M.P. Tooley ${ }^{1}$, \\ D.W. Grant ${ }^{1}$, D. Reboredo Gil ${ }^{1}$, P. Lepipas ${ }^{1}$, G. McKendrick ${ }^{1}$, S. Cipiccia ${ }^{1}$, S.M. Wiggins ${ }^{1}$, G. H. \\ Welsh $^{1}$, G. Vieux ${ }^{1}$, S. Chen ${ }^{1}$, C. Aniculaesei ${ }^{1}$, G.G. Manahan ${ }^{1}$, M-P Anania ${ }^{1}$, A. Noble ${ }^{1}$, S.R. Yoffe ${ }^{1}$, \\ G. Raj ${ }^{1}$, A. Subiel ${ }^{1}$, X. Yang ${ }^{1}$, Z.M. Sheng ${ }^{1}$, B. Hidding ${ }^{1}$, R.C. Issac ${ }^{1}$, M-H. Cho ${ }^{2}$, M.S. Hur ${ }^{2}$
}

${ }^{1}$ University of Strathclyde, Glasgow G4 0NG, Scotland, UK

${ }^{2}$ School of Natural Science, UNIST, Ulsan, 689-798, Korea

\begin{abstract}
Here we explore ways of converting laser radiation into coherent electromagnetic radiation using laser-driven plasma waves. Several schemes are presented, including colliding laser pulses in magnetized plasma and utilizing ultra-short electron bunches from laser wakefield accelerators to produce intense single-cycle pulses through coherent transition radiation and fewcycle coherent synchrotron radiation in undulators and plasma channels. These sources rely on high current electron bunches with femtosecond durations, which can result in radiation over a broad range of frequencies from 1 to $10^{5} \mathrm{THz}$.
\end{abstract}

\section{INTRODUCTION}

$\mathrm{P}$ LASMA is an extremely flexible medium for producing electromagnetic radiation. Here we focus on several methods for generating coherent radiation using plasma waves. A plasma density wake can be produced by the ponderomotive force of intense laser pulses travelling at speeds close to that of light in vacuum, which set in motion electron oscillations at the plasma frequency, thus creating a bubble-shaped accelerating structure (Fig. 1) that has a field gradient that can exceed 1 $\mathrm{TV} / \mathrm{m}$. We discuss how the ultra-short duration bunches of charged particles in laser wakefield accelerators (LWFAs) can be injected from the background plasma into the ion bubble and accelerate to 100 's $\mathrm{MeV}$ in millimetres [1-3].

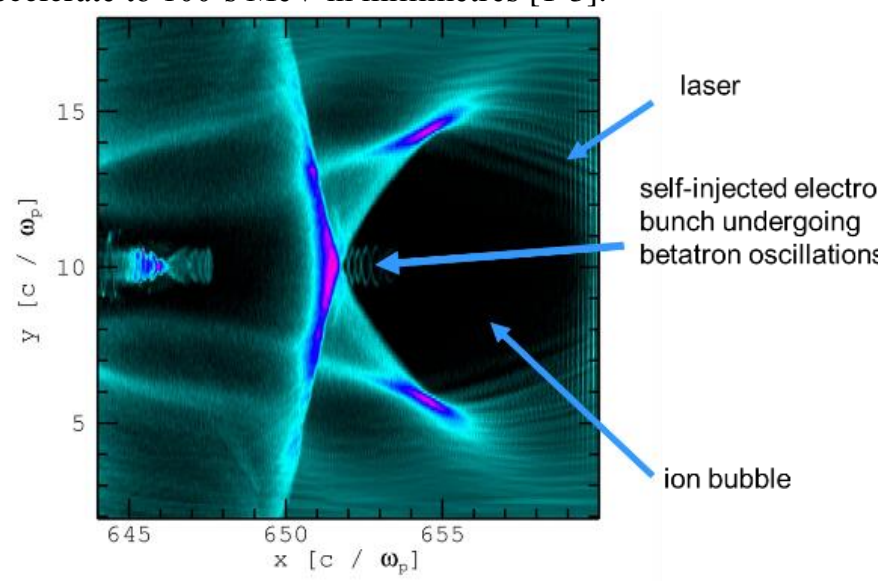

Fig. 1. LWFA bubble created by the ponderomotive force of the laser pulse. All lengths are normalized to $1 / \omega_{p}$, where $\omega_{p}$ is the plasma frequency.

Femtosecond duration bunches from a LWFA can lead to the emission of coherent radiation with high efficiency and over a broad spectral range stretching from $1 \mathrm{THz}$ to $10^{5} \mathrm{THz}$. Transverse acceleration of the ultra-short duration electron bunches in the bubble or an external undulator produces intense coherent synchrotron radiation. Intense single-cycle coherent transition radiation is also be produced when the femtosecond bunches pass through a thin metal foil.

A strong, localized, long-lasting electron oscillation in plasma also as a radiating antenna and emit continuous $\mathrm{THz}$ waves [4]. In this case the current is due to the ponderomotive force of two ultra-short duration, counter-propagating laser pulses in magnetized plasma. The field will grow if it is fed by the driving current while both electric and magnetic components of the field diffuse strongly and diffuse across the plasma-vacuum boundary, where they are converted to a propagating electromagnetic wave (Fig. 2).

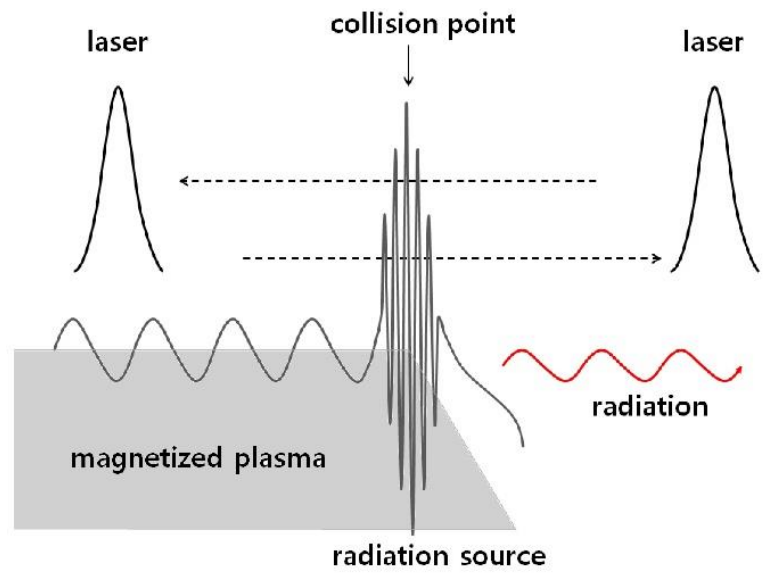

Fig. 2. THZ generation using colliding laser pulses in magnetised plasma [4].

Coherence of all beam-driven sources is governed by the spectral density of the electron bunch, $f(k)=\left|\int_{-\infty}^{\infty} e^{i k r} S(r) d r\right|^{2}$, where $S(r)$ is the density distribution and $k$ is the wavenumber of the radiation. Coherent radiation results when an ultra-short bunch is shorter than the wavelength of the emitted radiation, or the electron beam has a periodic density modulation, as in the free-electron laser (FEL) [5-7]. The power radiated coherently from $N$ bunched electrons is $P(k)=N(N-1) P_{1}(k) f(k)$, where $P_{1}(\mathrm{k})$ is the power radiated by a single electron [8]. For fully bunched beams $(f(k) \approx 1)$ the emission, $P(k) \approx N^{2} P_{1}$, is coherent [9]. This is the basis of the FEL, where the ponderomotive force of the radiation and undulator fields results in periodic bunching and $f(k) \rightarrow 1$. The coherent enhancement occurs at the resonant wavelength for the FEL and leads to efficient emission of radiation.

The laser wakefield accelerator (LWFA) accelerates electrons to high energies [1-3] using fields of evacuated 
plasma "bubbles" [10] created by the ponderomotive force of an intense laser pulse in plasma and the restoring Coulomb force of the ions [11]. In the "bubble regime" plasma electrons form a high-density sheath around the bubble, from which electrons are injected when their axial velocity exceeds that of the bubble [12] (Fig. 1). When the laser power exceeds the critical power for relativistic self-focussing the pulse compresses and increases the normalised vector potential $a_{0}$ of the laser field, which leads to injection of plasma electrons into the bubble. The injected electrons then accelerate until their Lorentz factor increases to $\gamma=\gamma_{d} \approx 2 \gamma_{g}^{2} a_{0} / 3$, at the dephasing length $L_{d}=4 c \gamma_{g}^{2} a_{0}^{1 / 2} / 3 \omega_{p}$, where $\gamma_{g}=\omega_{0} / \omega_{p}$, and $\omega_{p}$ and $\omega_{0}$ are the plasma and laser frequencies, respectively. The shortest bunches are injected close to threshold for self-injection, where injection is governed by charge build-up in the sheath crossing region at the back of the bubble [13]. The brief persistence of the charge build-up leads to femtosecond or sub-femtosecond duration electron bunches.

\section{RESULTS}

The experiments describe below have been carried out on the ALPHA-X [2, 14] beamline (Fig. 3), where 0.9 J, 40 fs, $800 \mathrm{~nm}$ laser pulses are focused to a $25 \mu \mathrm{m}$ (1/ $e^{2}$ radius) spot to give an initial intensity of $1.3 \times 10^{18} \mathrm{~W} / \mathrm{cm}^{2}\left(a_{0} \approx 0.8\right)$ on a He gas jet (Fig. 4), which ionises the gas to produce plasma with densities of $1-3 \times 10^{19} \mathrm{~cm}^{-3}$. Relativistic self-focussing results in a plasma bubble and a LWFA that produces $80-300 \mathrm{MeV}$ electron beams that can be very stable. The bunch charge is measured on a Lanex screen placed $0.6 \mathrm{~m}$ from the gas jet.

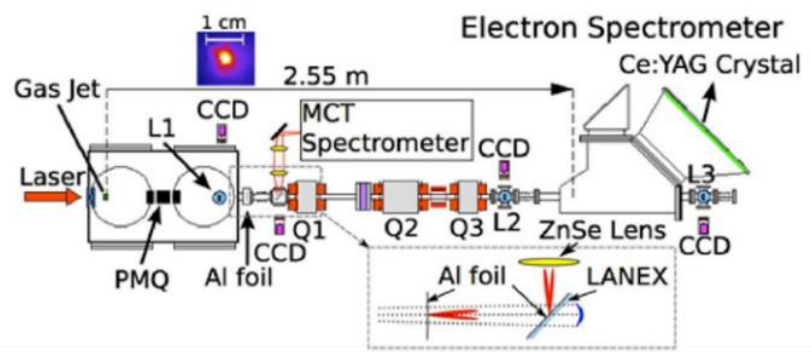

Fig. 3. ALPHA-X beamline used in the experiments.

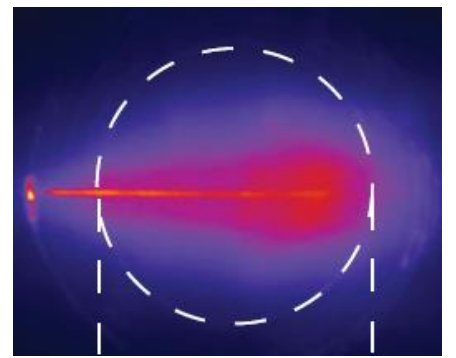

Fig. 4. Thomson scattered laser radiation from the plasma channel. The $2 \mathrm{~mm}$ diameter $\mathrm{He}$ gas jet is indicated by the dashed lines.

The LWFA is optimised for stable electron beam production (Figs. 5 - 7) close to threshold for injection. Electron beam energy spectra are measured using an imaging dipole magnet spectrometer placed $2.6 \mathrm{~m}$ from the gas jet. Two sets of triplet quadrupoles (PMQ and Q1, Q2, Q3) collimate the beam to optimise the resolution of the spectrometer (Fig. 3 and Fig. 5) and for transporting the beam through an undulator.

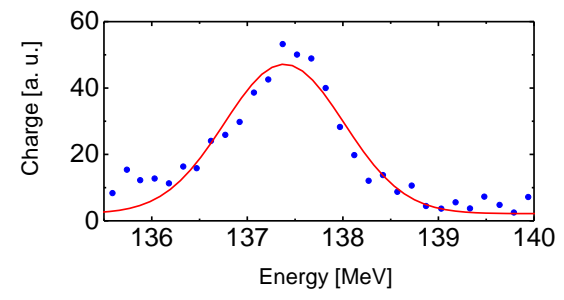

Fig. 5. Electron spectra $\left(\sigma_{\gamma} / \gamma=0.46 \%\right)$ for an electron beam for near threshold injection.

Electron beams with an emittance of $1 \pi \mathrm{mm} \operatorname{mrad}$ [15], energies in the range of $80-300 \mathrm{MeV}$, and percent level energy spreads [16] (Fig. 5), divergences of 1-2 mrad, peak currents $>1 \mathrm{kA}$ at the LWFA and femtosecond bunch durations [13] (Fig. 8), propagate through two coherent transition radiation (CTR) foils and then on to an undulator. Beam transport and CTR emission is modelled using GEANT4 for the experimental parameters.

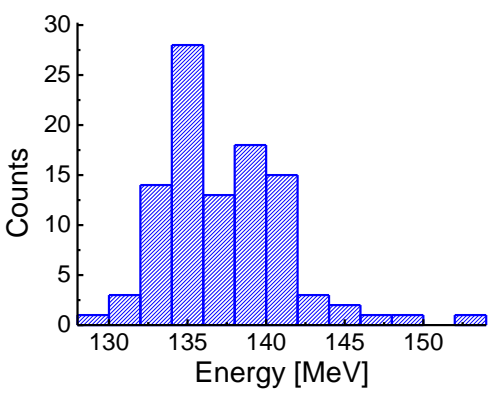

Fig. 6. Statistical distribution of the mean energy from the LWFA.

The pointing stability of the LWFA is excellent when the laser performance is optimised (Fig. 7), which allows comparison of simulated and measured CTR spectra. The CTR spectrum in Fig. 8, for electron energies of $90 \mathrm{MeV}$, has a peak around $4 \mu \mathrm{m}$, a dip around $7 \mu \mathrm{m}$ and a continuous rise to longer wavelengths.

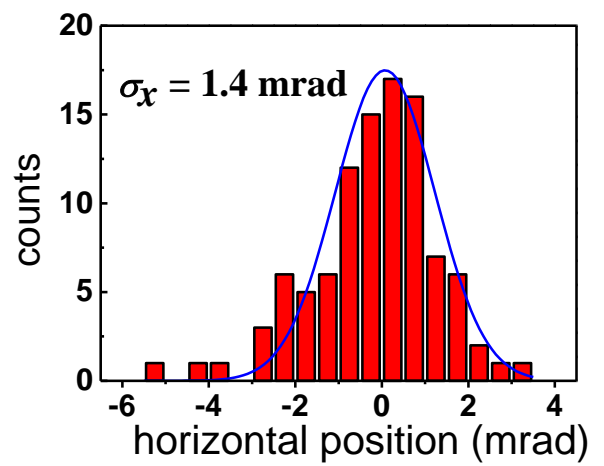

Fig. 7. Statistical distribution of LWFA pointing angle in the x-direction. A similar distribution is measured in the y-direction.

CTR spectra of smooth bunch shapes would show no structure. However, a train of two or more bunches can accurately reproduce the observed features: the dip at $7 \mu \mathrm{m}$ is consistent with two bunches separated by $11.5 \mathrm{fs}$ after $1 \mathrm{~m}$ propagation from the accelerator. The peak at $4 \mu \mathrm{m}$ requires at 
least one bunch to have a bunch duration at the source of $1 \mathrm{fs}$ for $2 \mathrm{mrad}$ r.m.s. divergence. The ratio of the amplitudes of the two peaks and the depth of the dip is reproduced when one bunch contains about $70 \%$ of a total charge of $10 \mathrm{pC}$. The short wavelength part of the spectrum is generated by the most energetic electron beams. The simulations shown in Fig. 9 have been obtained by setting the energy of two bunches to $100 \mathrm{MeV}$, close to the measured values.

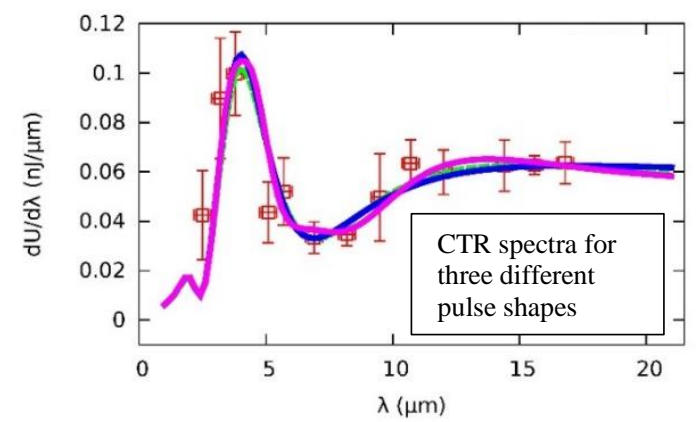

Fig. 8. Measured CTR spectra for $1 \mathrm{fs}$ bunches (at source) separated by $2 \mathrm{fs}$, but which then drift to $11.5 \mathrm{fs}$ at the CTR foils [13].

The measured spectra are consistent with the presence of additional $0.5 \mathrm{pC}$ ultra-short bunches with short inter-bunch delays. Fig. 9 shows the CTR spectra calculated from OSIRIS [17] PIC simulations, which indicate a strong peak around 4.5 $\mu \mathrm{m}$ due to multiple electron bunches with temporal separation of 2 fs and PIC Simulation-2 where there is no peak, which indicates the presence of a solitary bunch [13].

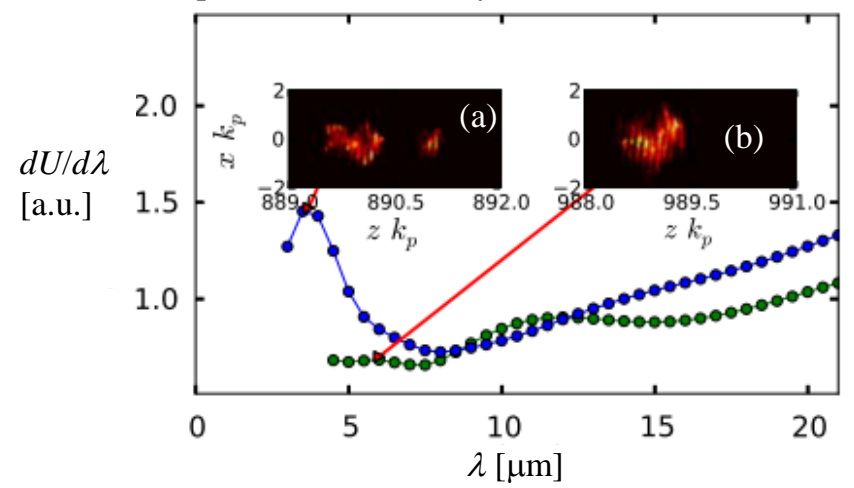

Fig. 9. Simulated spectra corresponding to Fig.2. Insets show bunch distributions of particle-in-cell (PIC) simulations using the OSIRIS PIC code [17] with experimental parameters for different bunch shapes (a) and (b) [13].

To demonstrate the LWFA as a source of synchrotron radiation the LWFA electron beam has been focussed through an undulator by matching the transport beta function with the undulator length using the quadrupole magnets. Initial experiments have been carried out in the visible using a $2 \mathrm{~cm}$ periodicity undulator [18] using $100 \mathrm{MeV}$ beams (using a laser at Jena). This has been replaced by a 100 period, $1.5 \mathrm{~cm}$ periodicity undulator with a slotted pole planar design and adjustable pole gap (at Strathclyde). The LWFA energy has also been increased to $130 \mathrm{MeV}$ (Fig. 10) to obtain XUV radiation (Fig. 11) [19] to explore the feasibility of a compact tuneable ultra-short pulse XUV synchrotron source. The distance from accelerator exit to undulator entrance is $3.5 \mathrm{~m}$ and undulator radiation spectra are measured using a calibrated VUV spectrometer and 16-bit CCD camera. The quadrupole lenses act as energy bandpass filters for electrons.

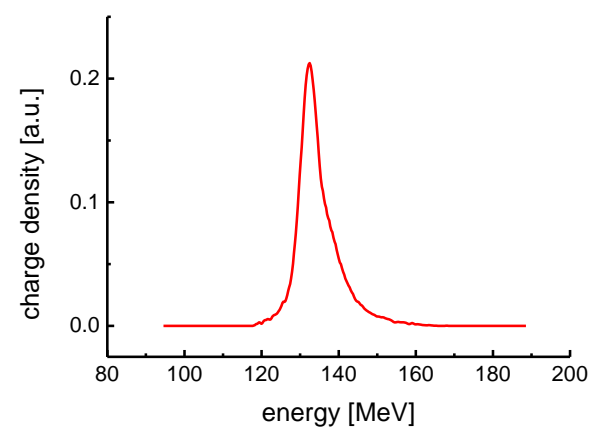

Fig. 10. Energy spectrum of LWFA beam used to produce undulator synchrotron radiation

The next stage in these investigations will be to optimise the beam transport to preserve the ultra-short bunch duration in the undulator so that $f(k) \approx 1$ for the resonant wavelength. This will ensure that the undulator radiation is coherent and will dispense with the need for FEL amplification, at least for frequencies in the range $1-200 \mathrm{THz}$ [9].

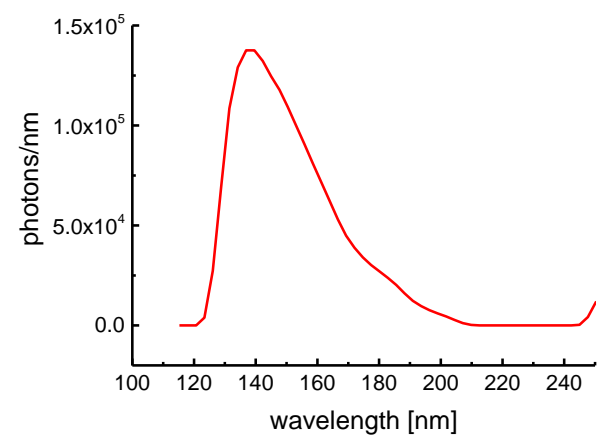

Fig. 11. VUV spectrum of radiation for 100 period, $1.5 \mathrm{~cm}$ period undulator for beam with energy spectrum in Fig. 10. A total of $10^{7}$ photons are measured.

The ion-channel laser (ICL) [20] is an ultra-compact alternative to the FEL. The ion channel, which acts as a compact undulator, can be formed by an ultra-short laser pulse or a relativistic charged particle bunch in plasma. ICL amplification can be described using a modified FEL formalism [20], which results in scaled equations for radiation and electron beam.

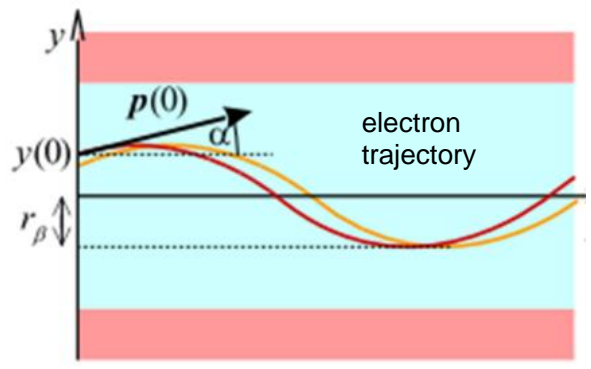

Fig. 12 Schematic of electron motion in an ion channel laser.

These equations suggest the feasibility of a compact coherent radiation source for wavelengths down to the XUV, with a gain parameter $\rho$ as high as 0.03 , which would give amplification for LWFA electron bunches with realistic energies and emittances.

In the ICL the undulation period depends on the ion density, electron energy and oscillation amplitude, in contrast with the 
FEL, where it is fixed by magnet spacing and periodicity. Similar to the FEL, the gain parameter is given by $\rho \approx 0.13\left[\left(\eta_{m} \eta_{f} / \gamma\right)\left(n_{b} / 10^{18} \mathrm{~cm}^{-3}\right)\left(R_{\beta} / \mu \mathrm{m}\right)^{2}\right]^{1 / 3}$, where the electron bunch density, $n_{b}=10^{16}-10^{20} \mathrm{~cm}^{-3}$, the betatron amplitude, $R_{\beta}=\left\langle\gamma_{0}^{1 / 2} r_{\beta}^{2}\right\rangle^{1 / 2} \gamma_{0}^{-1 / 4}=1-10 \mu \mathrm{m}$, for an electron beam Lorentz factor, $\gamma=10^{2}-10^{3}$, with coupling parameters $\eta_{m}=0.01-0.1, \eta_{f}=10^{-6}-0.1$, and $r_{\beta}$, the betatron amplitude [20]. With 100-200 MeV low energy spread electron beams from a LWFA (Fig. 5) [16] and low emittances [15], and high density bunches, $n_{b}=10^{20} \mathrm{~cm}^{-3}$, with the betatron amplitude $R_{\beta}=10 \mu \mathrm{m}$ and $\gamma=300$, the gain parameter $\rho=0.02$ for $\lambda>5 \mu \mathrm{m}$. [20]. For these parameters the betatron wavelength (which is the analogue of the undulator wavelength) $\lambda_{\beta}=\lambda_{p} \sqrt{2 \gamma}$ for a background plasma density of $1.8 \times 10^{19} \mathrm{~cm}^{-3}$ is $\approx 200 \mu \mathrm{m}$, which gives a gain length of $0.5 \mathrm{~mm}$, and a total length to saturation of the order of $1 \mathrm{~cm}$.

\section{CONCLUSION}

We have explored several unique coherent radiation sources based on laser-plasma interactions, some operational and some suggested. These can be quite intense and efficient sources and can produce very short duration pulses, down to a single cycle. Furthermore, they can be built around the same laser, which suggests that one could produce a very useful compendium of radiation sources for applications that require tunable ultrashort pulses for pump-probe time resolved studies.

We acknowledge the support of the UK EPSRC (grant no. $\mathrm{EP} / \mathrm{J} 018171 / 1$ ), the EU Extreme Light Infrastructure (ELI) project, Laserlab-Europe (no. 284464), and EUCARD-2 project (no. 312453). The authors would also like to thank the OSIRIS consortium (UCLA/IST) for the use of the PIC code OSIRIS. The authors are indebted to the Faculty of Engineering at the University of Strathclyde, for access to the High Performance Computer cluster. We also acknowledge the support of the Basic Science Research Program of the National Research Foundation (NRF) of Korea funded by the Ministry of Science, ICT and Future Planning (Grant number NRF2014M1A7A1A01030175 and NRF-2013R1A1A2006353).

The data associated with this research is available at doi: 10.15129/d130e97b-f080-48bc-aee7-1086f7e7091a

\section{REFERENCES}

J. Faure, Y. Glinec, A. Pukhov, S. Kiselev, S. Gordienko, E. Lefebvre, et al., "A laser-plasma accelerator producing monoenergetic electron beams," Nature, vol. 431, pp. 541-544, Sep 302004.

S. P. D. Mangles, C. D. Murphy, Z. Najmudin, A. G. R. Thomas, J. L. Collier, A. E. Dangor, et al., "Monoenergetic beams of relativistic electrons from intense laser-plasma interactions," Nature, vol. 431, pp. 535-538, Sep 2004.

C. G. R. Geddes, C. Toth, J. van Tilborg, E. Esarey, C. B. Schroeder, D. Bruhwiler, et al., "High-quality electron beams from a laser wakefield accelerator using plasma-channel guiding," Nature, vol. 431, pp. 538-541, Sep 302004.

M. H. Cho, Y. K. Kim, H. Suk, B. Ersfeld, D. A. Jaroszynski, and M. S. Hur, "Strong terahertz emission from electromagnetic diffusion near cutoff in plasma," New Journal of Physics, vol. 17, Apr 222015.
J. M. J. Madey, "Stimulated Emission of Bremsstrahlung in a Periodic Magnetic Field," Journal of Applied Physics, vol. 42, pp. 1906-\&, 1971.

R. Bonifacio, C. Pellegrini, and L. M. Narducci, "Collective Instabilities and High-Gain Regime in a Free-Electron Laser," Optics Communications, vol. 50, pp. 373-378, 1984.

D. A. Jaroszynski, P. Chaix, N. Piovella, D. Oepts, G. M. H. Knippels, A. F. G. vanderMeer, et al., "Superradiance in a shortpulse free-electron-laser oscillator," Physical Review Letters, vol. 78, pp. 1699-1702, Mar 1997.

J. S. Nodvick and D. S. Saxon, "Suppression of Coherent Radiation by Electrons in a Synchrotron," Physical Review, vol. 96, pp. 180184, 1954.

D. A. Jaroszynski, R. J. Bakker, A. F. G. Vandermeer, D. Oepts, and P. W. Vanamersfoort, "Coherent Startup of an Infrared FreeElectron Laser," Physical Review Letters, vol. 71, pp. 3798-3801, Dec 1993.

A. Pukhov and J. Meyer-ter-Vehn, "Laser wake field acceleration: the highly non-linear broken-wave regime," Applied Physics BLasers and Optics, vol. 74, pp. 355-361, Apr 2002.

T. Tajima and J. M. Dawson, "Laser Electron-Accelerator," Physical Review Letters, vol. 43, pp. 267-270, 1979.

S. V. Bulanov, F. Pegoraro, A. M. Pukhov, and A. S. Sakharov, "Transverse-wake wave breaking," Physical Review Letters, vol. 78, pp. 4205-4208, Jun 21997.

Islam R.M., E. Brunetti, R. P. Shanks, B. Ersfeld, R. Isaac, S. Cipiccia, et al., "Near-threshold electron injection in the laserplasma wakefield accelerator leading to femtosecond bunches," New Journal of Physics, in press New Journal of Physics, 2015.

D. A. Jaroszynski, R. Bingham, E. Brunetti, B. Ersfeld, J. Gallacher, B. van der Geer, et al., "Radiation sources based on laser-plasma interactions," Philosophical Transactions of the Royal Society aMathematical Physical and Engineering Sciences, vol. 364, pp. 689710, Mar 2006.

E. Brunetti, R. P. Shanks, G. G. Manahan, M. R. Islam, B. Ersfeld, M. P. Anania, et al., "Low Emittance, High Brilliance Relativistic Electron Beams from a Laser-Plasma Accelerator," Physical Review Letters, vol. 105, Nov 192010.

G. H. Welsh, S. M. Wiggins, R. C. Issac, E. Brunetti, G. G. Manahan, M. R. Islam, et al., "High resolution electron beam measurements on the ALPHA-X laser-plasma wakefield accelerator," Journal of Plasma Physics, vol. 78, pp. 393-399, Aug 2012.

R. A. Fonseca, L. O. Silva, F. S. Tsung, V. K. Decyk, W. Lu, C. Ren, et al., "OSIRIS: A three-dimensional, fully relativistic particle in cell code for modeling plasma based accelerators," Computational Science-Iccs 2002, Pt Iii, Proceedings, vol. 2331, pp. 342-351, 2002.

H. P. Schlenvoigt, K. Haupt, A. Debus, F. Budde, O. Jackel, S. Pfotenhauer, et al., "A compact synchrotron radiation source driven by a laser-plasma wakefield accelerator," Nature Physics, vol. 4, pp. 130-133, Feb 2008.

M. P. Anania, E. Brunetti, S. M. Wiggins, D. W. Grant, G. H. Welsh, R. C. Issac, et al., "An ultrashort pulse ultra-violet radiation undulator source driven by a laser plasma wakefield accelerator," Applied Physics Letters, vol. 104, Jun 302014.

B. Ersfeld, R. Bonifacio, S. Chen, M. R. Islam, P. W. Smorenburg, and D. A. Jaroszynski, "The ion channel free-electron laser with varying betatron amplitude," New Journal of Physics, vol. 16, Sep 222014. 